\title{
Analysis of Disturbance Properties of Solar Array Drive Assembly in Different Sub-Divisions Drive
}

\author{
Mariyam Sattar ${ }^{1, *}$, Cheng Wei ${ }^{1}$, Jiangpan Chen $^{1}$ and Asif Israr ${ }^{2}$ \\ ${ }^{1}$ School of Aeronautic Science \& Engineering, Beihang University, Beijing 100191, Haidian District, China \\ ${ }^{2}$ Department of Mechanical Engineering, 1 Islamabad Highway, P O Box 2750, Islamabad-4000, Pakistan \\ Corresponding author
}

\begin{abstract}
The micro-vibrations, generated by Solar Array Drive Assembly (SADA), of satellite can extensively alter and reduce its imaging quality as well as pointing direction precision. The present work aims to analyze the active and structural vibration disturbances of SADA driven in two different kinds of subdivision number (32 and 64) through theoretical analysis and experimentation. The considered drive mechanism is a two phase and four beat hybrid stepper motor. The formulations to calculate frequencies, forces and moments demonstrated by SADA, operating with various sub-division numbers, are derived leading to its comprehensive disturbance model. The developed mathematical expressions contain information related to period of input of electric pulse signal, solar array speed, number of rotor teeth, reduction ratio, amplitude coefficients, phase of disturbance force, beat number and sub-divisions. In order to validate the theoretical results, amplitude of disturbance forces and moments, produced by SADA driving no load / dead load, is acquired by using a rigid force / moment test platform. The obtained results verify that increase in sub-division number from 32 to 64 leads to reduction in disturbance force and moment generated by SADA, enhances its stability and smoothen the drive. The research work can find good deal of its applicability for drive circuit designing and vibration damping study of SADA
\end{abstract}

Keywords-micro-vibrations; solar array drive assembly (sada); active vibration; structural vibration; sub-division number; beat number; reduction ratio

\section{INTRODUCTION}

The micro-vibrations of satellite have characteristics of reduced amplitude \& widespread frequency range so, these can significantly reduce its aiming accuracy and imaging quality [1]. These micro-vibrations are generally generated due to the static and dynamic unbalanced forces of rotary components on satellites [2]. There are many possible disturbance sources, for example RWAs (Reaction Wheel Assemblies), CMGs (Control Moment Gyros) and SADA [3]. The disturbances of RWAs and CMGs have been studied for a long time, and many meaningful results have been achieved. However, the study on SADA disturbance is sparse and a very little literature targeting on analysis of disturbance properties of SADA driving dead, rigid and flexible load can be found [4-6]. So, with the development of high precision satellite it is imminent to research on various modes of SADA disturbance. The key performance of SADA is to ensure the normal of solar array is parallel to the solar beam. It is done by driving the solar panels that keep the track of solar emission and help spacecraft get sun energy as much as possible. In addition, SADA also transmits signals and transfers energy to satellite. In some kinds of satellites, solar array and its drive assembly is used not only in energy system and signals transmission but also as an actuator to control synchronous satellite attitude [7]. SADA usually uses stepper motor as a driver. It is used to transform incoming electric pulses and get output in the form of angular displacement or linear displacement. Moreover, it has the advantages of high positioning accuracy, no cumulative error and simple open-loop control [8]. However, input of every electrical pulse signal will produce an impact load on SADA and this is one of its major disturbance sources. At present, subdivision drive (SDD), which can improve the smoothness, stability, positioning accuracy, eliminates low torque ripples, reduce resonance effects and improve resolution of SADA by reducing the step angle of stepper motor, is frequently used [9]. The reduction of step angle is completed by changing the input current from traditional rectangular wave current into ladder wave current, means, a complete electric pulse signal will be decomposed into several micro electric pulses [10]. Even after decomposition of a full signal and using micro-step drive, the disturbances induced by SADA still exist and this time these are generated by input of micro electric pulse signals. However, the disturbance spectral line after subdivision is sparser than before subdivision in both frequency and time domain.

The stepper motor researched in this paper is a four-beat \& two-phase hybrid stepper motor. The disturbance characteristics of SADA, driven in two different kinds of subdivision (SD) number (32 and 64), are obtained through theoretical analysis and experimentation. The outline of this paper is detailed as; section II provides mathematical SADA disturbance model considering active and structural vibration disturbances, its response in several different conditions is tested by using a rigid force / moment test platform in section III, a comprehensive discussion on results and comparison between 32 \& 64 subdivisions drive is provided next, followed by conclusion and future recommendations. The results in this work can help in improving the design of SADA drive circuits and its vibration attenuation study.

\section{SADA DISTURBANCE}

There are two main causes of disruptions induced by SADA namely active and structural vibration disturbances. Active vibration disturbance is caused by stepper motor's input of electric pulse signal. Structural vibration disturbance can be further categorized into electromagnetic coupling disturbance and structural coupling disturbance. The parameters of SADA used in this section are presented in Table 1. 
TABLE I. SADA PARAMETERS

\begin{tabular}{ccc}
\hline Parameter & Physical meaning & Value \\
\hline$\omega_{0}$ & Solar array speed & $7.27 \times 10^{-5} \mathrm{rad} / \mathrm{s}$ \\
\hline$q$ & Reduction ratio & 100 \\
\hline$Z$ & Number of rotor teeth & 50 \\
\hline$p$ & Number of beats & 4 \\
\hline$n$ & Number of subdivisions & $32 / 64$ \\
\hline$I$ & Current amplitude & $0.1 \mathrm{~A}$ \\
\hline$K_{t}$ & $\begin{array}{c}\text { Coefficient of electromagnetic } \\
\text { torque }\end{array}$ & $4 \mathrm{~N} \cdot \mathrm{m} / \mathrm{A} \cdot \mathrm{rad}$ \\
\hline$J$ & Moment of inertia of load & $25 \mathrm{~kg} \cdot \mathrm{m}^{2}$ \\
\hline
\end{tabular}

\section{A. Active Vibration Disturbance}

As discussed above, the induced electrical pulse signal, which is characterized by period, will produce an impact load on SADA. This period is associated to the rotor speed and number of rotor teeth, beats \& subdivisions. The rotor speed is related to the solar array speed and reduction ratio. The equation of the period and the rotor speed is;

$$
\begin{array}{r}
T=\frac{\frac{2 \pi}{\omega}}{n p z} \\
\omega=\omega_{0} \times q
\end{array}
$$

where, $\omega$ is the rotor speed, and $T$ is the input period of the electric pulse signal. Substituting "(2)" in "(1)”, the function of input period can be written as;

$$
T=\frac{2 \pi}{\omega_{0} q n p z}
$$

Therefore, the fundamental frequency is given by;

$$
f_{0}=\frac{1}{T}=\frac{n p z \omega_{0} q}{2 \pi}
$$

As the input of electric pulse signal is characterized by period in time domain, the disturbance frequency is discrete in frequency domain, and the disturbance frequencies can be written as;

$$
f=\sum_{i=1}^{\infty} i f_{0}
$$

Substituting the parameters in “(4)”, SADA active vibration disturbance frequencies running in 32 and 64subdivision can be written as;

$$
f_{32}=\sum_{i=1}^{\infty} 7.4 i \quad ; \quad f_{64}=\sum_{i=1}^{\infty} 14.8 i
$$

where $f_{32}$ and $f_{64}$ are active vibration disturbance frequencies of SADA running in 32-subdivision and 64-subdivision respectively.

\section{A. Structural Vibration Disturbance}

Structural vibration disturbance can be mainly divided into two parts. One part is electromagnetic coupling disturbance that is produced because of presence of electromagnetic stiffness. When the stepper motor is powered, due to the effect of electromagnetic torque, electromagnetic torsion stiffness will be generated between the stator and rotor. When the stepper motor works driving load, it can be simplified as a system with torsion spring - moment of inertia and the natural frequency of this system is named electromagnetic frequency [11]. Reference [11, 12] shows the solving formula of the electromagnetic frequency and electromagnetic stiffness, which is;

$$
f_{e m}=\frac{1}{2 \pi} \sqrt{\frac{q K_{e m}}{J}} ; K_{e m}=K_{t} I p
$$

where $f_{e m}$ is the electromagnetic frequency, and $\mathrm{K}_{\mathrm{em}}$ is the electromagnetic stiffness. When SADA run without load, the moment of inertia is extremely small and the electromagnetic frequency will be very large. In order to test the characteristics of the electromagnetic frequency, SADA disturbance running with a rigid load will be tested.

Substituting the parameters in "(7)", the electromagnetic frequency and electromagnetic stiffness running with a rigid load can be obtained as;

$$
f_{\text {em }}=1.42 \mathrm{~Hz} ; \quad K_{e m}=20 \mathrm{~N} \cdot \mathrm{m} / \mathrm{rad}
$$

The other part is structural coupling disturbance. As discussed in section "A", every input of the electric pulse signal will product an impact on SADA, whose frequency band is infinite. Therefore, it results in all of the natural frequencies of the entire mechanic system to get activated, and when the natural frequency is near or identical to the disturbance frequency of active vibration, the disruption will be dramatically magnified. As, SADA active vibration disturbance frequency for 32 subdivisions drive is $7.4 \mathrm{~Hz}$ and for 64 subdivisions drive it is $14.8 \mathrm{~Hz}$ or their multiples, so, the natural frequencies near to or falling in these ranges will intensify corresponding disturbance response.

\section{B. SADA Disturbance Model}

Taking all the disturbances into account, SADA disturbance model running in different subdivision number can be written as;

$$
\left\{\begin{array}{l}
m_{32}(t)=\sum_{i=1}^{n} C_{i} \sin \left(14.8 i \pi+\alpha_{i}\right)+C_{e m} \sin \left(2 \pi f_{e m}+\alpha_{e m}\right)+\sum_{j=1}^{k} C_{j} \sin \left(2 \pi f_{j}+\alpha_{j}\right) \\
m_{64}(t)=\sum_{i=1}^{n} C_{i} \sin \left(29.6 i \pi+\alpha_{i}\right)+C_{e m} \sin \left(2 \pi f_{e m}+\alpha_{e m}\right)+\sum_{j=1}^{k} C_{j} \sin \left(2 \pi f_{j}+\alpha_{j}\right)
\end{array}\right.
$$

where $m_{32}(t)$ and $m_{64}(t)$ are disturbance force in 32subdivision and in 64-subdivision drive respectively, $C_{i}, C_{e m}$ and $C_{j}$ are the amplitude coefficients of the disturbance force, and $\alpha_{i}, \alpha_{e m}$ and ${ }^{\alpha_{j}}$ are the phases of the disturbance force. 


\section{EXPERIMENTAL SETUP / TEST PlATFORM}

This experiment is completed in a force/moment testing platform as shown in Figure 1. This platform consists of eight unidirectional piezoelectric sensors. There are four sensors placed in the horizontal direction in order to test the two horizontal disturbance forces and the vertical disturbance torque. The other four sensors are placed in the vertical direction in order to test the two horizontal disturbance moments and the vertical disturbance force. The inside of the platform is empty, so the measured object can be installed either inside or outside the platform. This platform is rigid and its fundamental frequency is higher than $500 \mathrm{~Hz}$. The measuring accuracy of the disturbance force and moment is more than $0.001 \mathrm{~N}$ and $0.001 \mathrm{~N} \cdot \mathrm{m}$ respectively.

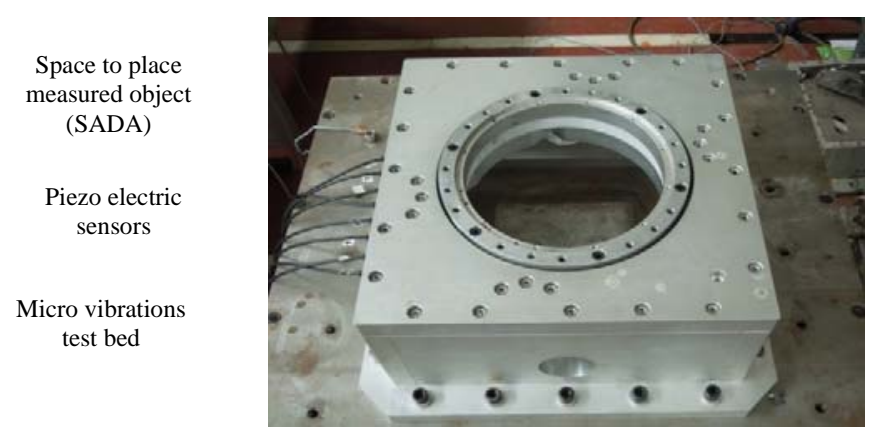

FIGURE I. TESTING PLATFORM

(a)

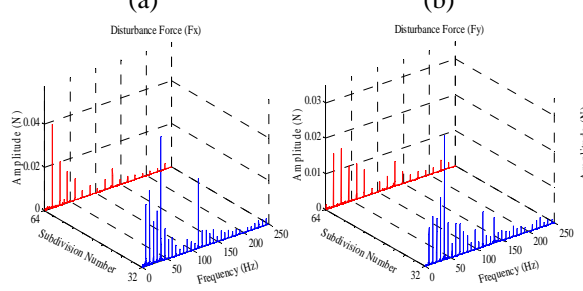

(c)

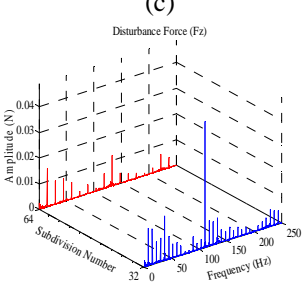

(d)

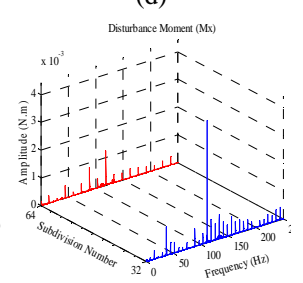

\section{RESULTS AND DisCUSSION}

The sample frequency of this experiment is $2048 \mathrm{~Hz}$, and the sample time is $16 \mathrm{~s}$. Disturbance forces \& moments in frequency domain $(0-250 \mathrm{~Hz})$ \& time domain $(0-16 \mathrm{~s})$ are shown in the form of plots. In order to show the test results clearly, the results for amplitude is given the same axis when SADA runs with identical load but in different SD numbers. The comparison is shown in Figure II \& III.

Figure II from (a) to (f) shows SADA running in different SD numbers without load in frequency domain. In 32-SDD the highest amplitude, of frequency, for disturbance force Fx \& Fy, is observed below $50 \mathrm{~Hz}$, while it lies in between $100 \mathrm{~Hz}-$ $150 \mathrm{~Hz}$ for rest of them. For 64-SDD response for disturbance forces lie in between 0 to $50 \mathrm{~Hz}$ and between $100 \mathrm{~Hz}-150 \mathrm{~Hz}$ for disturbance moments. Moreover, the amplitude of disturbance for later is far lesser when compared with 32-SDD. When there is input of electric pulse signal SADA moves to next position but instead of staying at equilibrium it starts oscillating about it. For 32-SDD, there are 7.4 signals in one second in comparison to 14.8 signals for 64-SDD. The angle between two steps for 32-SDD is larger providing bigger oscillation or resonance zone. Within this zone, whenever excitation \& natural frequencies coincide, very high amplitude is observed. 64-SDD doesn't get enough roam \& time to oscillate \& interfere with structure natural frequency, in resonance zone, giving reduced amplitudes of disturbance.

FIGURE II. SADA RUNNING IN DIFFERENT SUBDIVISION NUMBER WITHOUT LOAD IN FREQUENCY DOMAIN (a) Fx (b) Fy (c) Fz (d) Mx (e) My (f) $\mathrm{Mz}$

The number of frequencies excited by SADA with 32-SDD is more as compared to 64-SDD. Active vibration disturbance frequencies for 64-SDD are multiple of $14.8 \mathrm{~Hz}$ while for 32SDD these are multiple of either $7.4 \mathrm{~Hz}$ or $14.8 \mathrm{~Hz}$. So, 32SDD excites the structure natural frequencies more frequently. Thus, larger number of excitation frequencies in 32-SDD get chance to coincide with structure natural frequencies leading to more frequent disturbance behavior causing degradation in performance.

Amplitude of disturbance frequencies decrease as one move towards higher frequency domain. If frequency domain from $200 \mathrm{~Hz}-250 \mathrm{~Hz}$ is observed, it is clear that disturbance amplitude in this range for SADA with 64 SD have almost died out but 32-SDD is still showing pronounced value of disturbance frequency amplitude. Thus, even in higher frequency domain (range considered for analysis) SADA with 32 SD can cause the attached structure to exhibit vibration with dangerously large amplitude.
Figure III (a) to (f) shows the amplitude (N / Nm) of disturbance force and moment when SADA with different SD numbers is operated for duration of $16 \mathrm{~s}$. Disturbance response for SADA with SD 64 lies in a narrow range about the mean position with maximum positive \& negative amplitudes not exceeding $0.15(\mathrm{~N} / \mathrm{Nm})$. A pronounced difference in amplitudes is observed when response trend for SADA with SD 32 is observed clearly depicting the micro instabilities and bigger height of amplitude induced by this SDD.

The overall comparison of plots in figure II \& III show that SADA with SD number 64 is more preferable because of less number of excited frequencies, very reduced amplitude of excited frequencies thus ensuring smooth \& noiseless drive and either less or almost no excited frequencies (Figure II) as one proceeds towards higher frequency domain. 
(a)

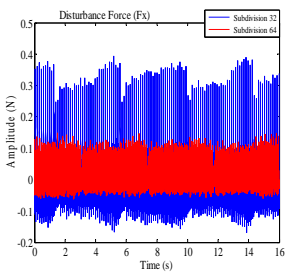

(b)

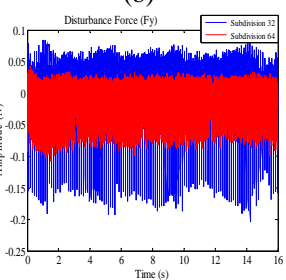

(c)

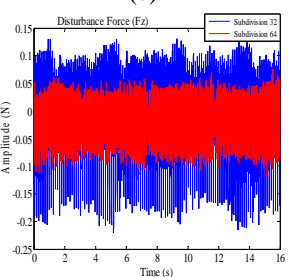

(d)

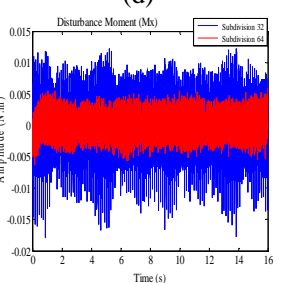

(e)

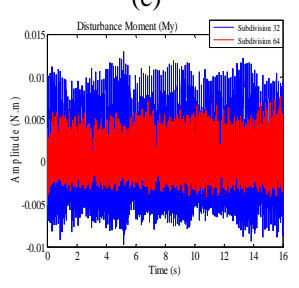

(f)

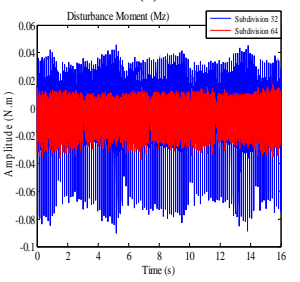

FIGURE III. SADA RUNNING IN DIFFERENT SUBDIVISION NUMBER WITHOUT LOAD IN TIME DOMAIN (a) Fx (b) Fy (c) Fz (d) Mx (e) My (f) Mz

Table II shows the numerical data and Figure IV shows graphical explanation for comparison of average of disturbance forces/moments in, $\mathrm{x}, \mathrm{y} \& \mathrm{z}$-direction, generated by SADA with different SDD.

TABLE II. AVERAGE OF DiStURBANCE ForCES AND MOMENTS AMPLITUDES

\begin{tabular}{ccc}
\hline Disturbance force/moment & 32-Subdivision & 64-Subdivision \\
\hline$F x(N)$ & $7.77 \mathrm{E}-02$ & $3.69 \mathrm{E}-02$ \\
\hline$F y(N)$ & $4.36 \mathrm{E}-02$ & $2.28 \mathrm{E}-02$ \\
\hline$F z(N)$ & $5.08 \mathrm{E}-02$ & $2.39 \mathrm{E}-02$ \\
\hline$M x(N \cdot \mathrm{m})$ & $4.00 \mathrm{E}-03$ & $1.79 \mathrm{E}-03$ \\
\hline$M y(N \cdot \mathrm{m})$ & $3.66 \mathrm{E}-03$ & $1.54 \mathrm{E}-03$ \\
\hline$M z(N \cdot \mathrm{m})$ & $1.53 \mathrm{E}-02$ & $6.10 \mathrm{E}-03$
\end{tabular}

Disturbance values for SADA with SD 32 are more than double of the ones generated by SADA with SD 64. As the subdivision number is reduced the stator flux is not able to move smoothly leading to increased oscillations and instabilities. SADA with SD 64 can improve system performance because smaller step angles lead to better positioning \& almost diminish oscillations.

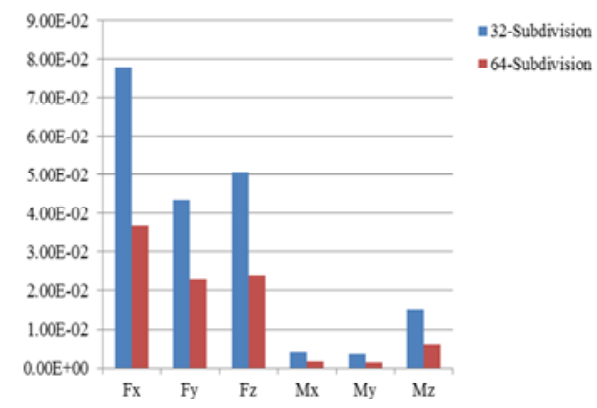

FIGURE IV. AMPLITUDE (N \& Nm) COMPARISON THROUGH BAR CHART

\section{CONCLUSION AND FUTURE GOALS}

Theoretical formulation and experimentation is completed to research the disturbance properties of SADA driven in two different kinds of subdivisions i.e. 32 \& 64. Analysis of disturbances forces and moments reveal that SADA with subdivision number 64 is more preferable to drive solar array panels mounted on spacecraft because they present a very smooth drive leading to highly accurate performance. Authors are working to make improvements in the experimental setup by addition of gravity unload device to simulate the working environment in space (zero gravity conditions). This gravity unload device consist of linear bearing, air bearing and a rigid holder. Experimentation on SADA, with different subdivision numbers, running with \& without gravity load is underway. Working will be completed to determine the effect of $1 \mathrm{G}$ and zero $G$ on amplitude of disturbance torque, electromagnetic and active vibration disturbance frequencies of SADA with various SD. The designed experimental setup will not only reduce the launch and operation costs but also provide help in practical demonstration (on ground) to disturbance study of SADA during in-orbit operations.

\section{REFERENCES}

[1] Luo, Q., et al., "Dynamic modelling and observation of micro-vibrations generated by a Single Gimbal Control Moment Gyro", Journal of Sound and Vibration, 2013. 332(19): p. 4496-4516.

[2] Masterson, R.A., "Development and validation of empirical and analytical reaction wheel disturbance models", 1999, Massachusetts Institute of Technology.

[3] Wei, Z.P.C. and Z. Yu, "Measure of reaction wheels disturbance considering coupling effect", Journal of Beijing University of Aeronautics and Astronautics, 2011. 8: p. 008.

[4] Chen, J.P., W. Cheng, and W. Han. "Analysis and Simulation of Stepper Motor Disturbance Considering Structural Coupling", Applied Mechanics and Materials. 2014. Trans Tech Publ.

[5] Chen, J.P., W. Cheng, and Y.F. Wang. "Modeling and Simulation of Solar Array Drive Assembly Disturbance Driving a Flexible Load", Applied Mechanics and Materials. 2014. Trans Tech Publ.

[6] Li, X., W. Cheng, and W. Han, "Disturbance modeling, simulation and testing of solar array drive assembly", Int J Hybrid Inform Technol, 2014. 7: p. 123-137.

[7] Xing, G. and W. Youping, "The universalization-, serialization-, and modularization design of solar array drive assembly (SADA)". Chin. J. Space Sci, 2002. 22: p. 55-67.

[8] ZHANG, M., et al., "A high stability control method for solar array drive mechanism", Aerospace Control and Application, 2010. 4: p. 010.

[9] Baluta, G. "Microstepping mode for stepper motor control", International Symposium on Signals, Circuits and Systems. 2007.

[10] Dingbang, L., "Two phase hybrid stepping motor subdivision drive" 2012, Changsha University of Science \& Technology: B.E.( HuNan University of Technology). p. 44.

[11] Yang, Y.L., et al. "Experiment and simulation of electromagnetic stiffness for stepper motor", Applied Mechanics and Materials. 2010. Trans Tech Publ.

[12] Bodson, M., J.S. Sato, and S.R. Silver, "Spontaneous speed reversals in stepper motors", Control Systems Technology, IEEE Transactions on, 2006. 14(2): p. 369-373. 